ANALYZING POWER MEASUREMENTS IN HIGH-P ${ }_{\perp}^{2}$ p-p ELASTIC SCATTERING*

R.S. Raymond, K.A. Brown, R.J. Bruni, P.R. Cameron,

D.G. Crabb, R.L. Cummings, F.Z. Khiari, A.D. Krisch, A.M.T. Lin, K.M. Terwilliger

Randall Laboratory of Physics, The University of Michigan Ann Arbor, Michigan 48109

G.T. Danby, Y.Y. Lee, L.G. Ratner

Accelerat or Department, Brookhaven National Laboratory Upton, NY 11973

\title{
J.R. O'Fallon
}

Office of Advanced Studies and Department of Physics, University of Notre Dame, Notre Dame, Indiana 46556

\section{D.C. Peaslee}

Department of Physics, University of Maryland

College Park, Maryland 20742

T.S. Bhatia, G. Glass, L.C. Northcliffe

Department of Physics, Texas A \& M University

College Station, Texas 77843

\section{ABSTRACT}

The analyzing power in $28 \mathrm{GeV} / \mathrm{c}$ proton-proton elastic scattering was measured at $\mathrm{P}_{\perp}^{2}=5.95$ and $6.56(\mathrm{GeV} / \mathrm{c})^{2}$ using a polarized proton target and an unpolarized proton beam at the Brookhaven National Laboratory AGS. Results indicate that the analyzing power, $A$, is rising sharply with $\mathrm{P}_{\perp}^{2}$.

Previous measurements ${ }^{1}$ of the analyzing power, A, in $\mathrm{p}+\mathrm{p} \rightarrow$ $p+p$ suggested a rise in $A$ at large $-P^{2}$, but the statistical uncertainty in the highest point at $\mathrm{P}^{2}=5.95(\mathrm{GeV} / \mathrm{c})^{2}$ made it impossible to determine the magnitude of the increase. In an effort to clarify this situation, we made new measurements of $A$ at $\mathrm{P}_{1}^{2}=$ 5.95 and $6.56(\mathrm{GeV} / \mathrm{c})^{2}$.

An unpolarized beam of typically $5 \times 10^{10} 28 \mathrm{GeV} / \mathrm{c}$ protons from the AGS at Brookhaven National Laboratory was incident upon the University of Michigan polarized proton target. This target contains irradiated ammonia beads cooled to $0.5^{\circ} \mathrm{K}$ by a ${ }^{3} \mathrm{He}-{ }^{4} \mathrm{He}$ evaporation refrigerator, in a $2.5 \mathrm{~T}$ magnetic field. The polarizing transitions are driven by a $70 \mathrm{GHz}$ microwave system. The polarization of the hydrogen protons is measured with a $107 \mathrm{MHz}$ NMR system, and is typically $\mathrm{P}_{\mathrm{T}}=45 \%$ with beam and $60 \%$ without beam.

*Work performed under the auspices of the U.S. Department of Energy. 
Elastic proton events were detected in the double-arm forwardbackward spectrometer shown in Fig. 1 consisting of six magnets and six scintillation counter hodoscopes with a $\mathrm{P}_{\perp}^{2}$ acceptance of about $1(\mathrm{GeV} / \mathrm{c})^{2}$.

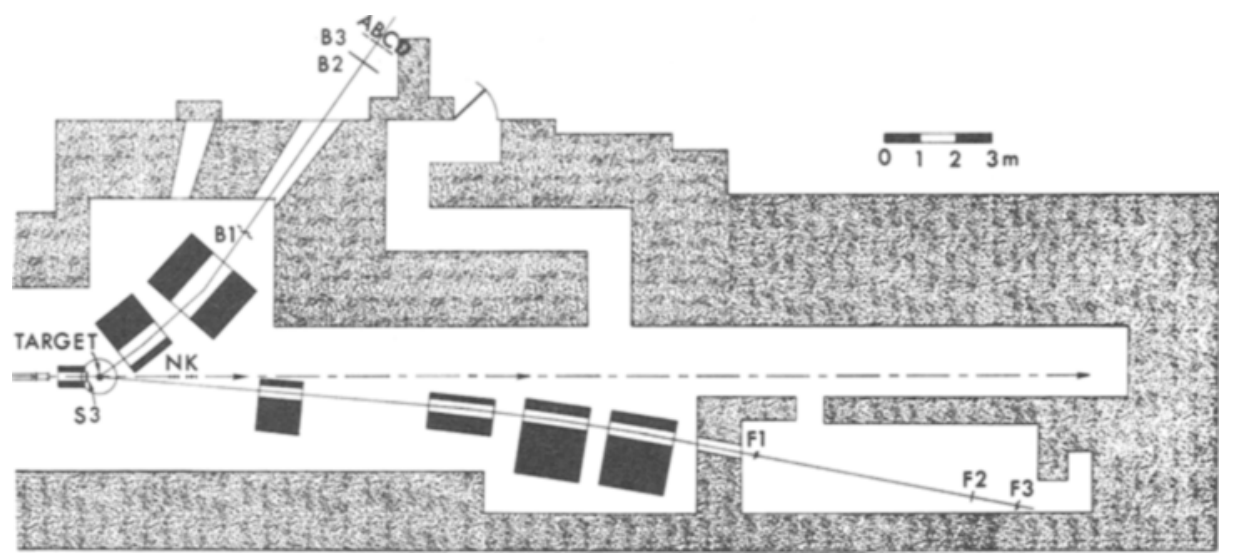

Fig. 1. Double-arm forward-backward spectrometer.

The analyzing power for these measurements is obtained, following the Basel convention, from

$$
A=-\frac{1}{P_{T}} \frac{N(t)-N(t)}{N(t)+N(t)}
$$

where $N(\uparrow)$ and $N(t)$ are the numbers of events with the target polarization up and down, respectively. The new values for $A$ are:

$$
\begin{array}{cc}
\mathrm{P}_{\perp}^{2}\left[(\mathrm{GeV} / \mathrm{c})^{2}\right] & \mathrm{A} \\
\perp 5.95 & 16 \pm 5.7 \\
6.56 & 51 \pm 17
\end{array}
$$

The point at $\mathrm{P}_{\perp}^{2}=5.95(\mathrm{GeV} / \mathrm{c})^{2}$ includes our earlier data. ${ }^{2}$ These points are plotted in Fig. 2 along with earlier data from the AGS ${ }^{1,2}$ and CERN. ${ }^{3}$ Notice the sharp increase in A near $P_{\perp}^{2}=6$ $(\mathrm{GeV} / \mathrm{c})^{2}$, which was totally unexpected. 


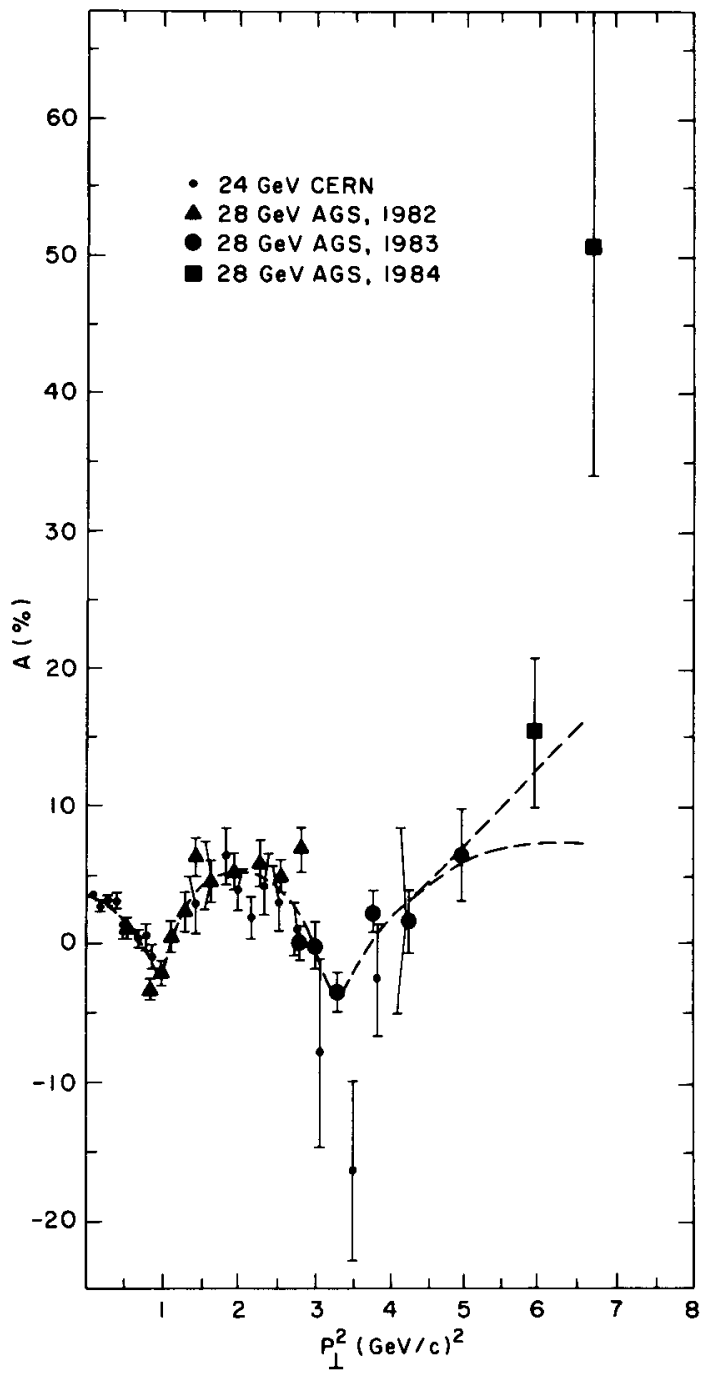

Fig. 2. Analyzing power for proton-proton elastic scattering as a function of $\mathrm{P}^{2}$.

\section{REFERENCES}

1. D.C. Peaslee et al., Phys. Rev. Lett. 51, 2359 (1983).

2. P.H. Hansen et al., Phys. Rev. Lett. $5 \overline{0,} 802$ (1983).

3. J. Antille et al., Nucl. Phys. B185, $\overline{1}$ (1981). 